\title{
Primer registro de la especie Myotis chiloensis (Waterhouse, 1838) (Chiroptera, Vespertilionidae) en el Parque Nacional Alberto de Agostini (Región de Magallanes y Antártica Chilena)
}

\author{
First record of Myotis chiloensis (Waterhouse, 1838) (Chiroptera, Vespertilionidae) \\ at the Alberto de Agostini National Park (Magellan region and Chilean Antarctica, Chile)
}

Gonzalo Ossa ${ }^{1,2}$

De las trece especies de quirópteros que se distribuyen dentro del territorio nacional (Díaz et al. 2011; Ossa et al. 2014; Rodríguez-San Pedro et al. en preparación), sólo tres han sido descritas para la región de Magallanes y Antártica Chilena, estas especies corresponden a Lasiurus varius (Poeppig, 1835), Histiotus magellanicus (Philippi, 1866) y Myotis chiloensis (Waterhouse, 1840) (Dabbene, 1902; Martinic, 1957; Koopman, 1967; Texera, 1973; Mann, 1978; Rau \& Yáñez, 1979; Massoia \& Chebez 1993; Venegas \& Sielfeld 1998; Ossa \& Díaz, 2014;). Estas especie son aquellas que presentan la distribución más austral en el mundo (Koopman, 1967; Giménez et al. 2012; Ossa \& Díaz, 2014), sin embargo existen pocos estudios en la región, y la totalidad de registros han sido obtenidos en zonas pobladas como Punta Arenas, Puerto Navarino o Puerto Natales.

Myotis chiloensis es un murciélago insectívoro pequeño, nativo del extremo sur de Sudamérica (Ossa et al. 2010). En Chile se distribuye desde Coquimbo hasta Isla Navarino, utilizando tanto zonas de bosque como zonas urbanas para alimentarse (Ossa \& Rodríguez-San Pedro, 2015). Se alimenta únicamente de insectos que captura al vuelo, como Nematoceros y Tipulidos (Koopman, 1967). Posee una alta maniobrabilidad en vuelo y llamadas de ecolocación que le permiten moverse fácilmente dentro de zonas boscosas densas (Bozinovic et al. 1985; Ossa et al. 2010). Posee un pelaje relativamente largo y bicoloreado, siendo más oscuro en la base y más claro en las puntas (Díaz et al. 2011). Es la única especie del género Myotis conocida en estas latitudes (Mann,
1978; Iriarte, 2008). Puede ser confundido con la especie H. magellanicus (Ossa \& Díaz, 2014) dado que el color de su pelaje es similar, pero $M$. chiloensis posee orejas más cortas, que no superan los $12 \mathrm{~mm}$ desde la base hasta la punta, en cambio H. magellanicus posee orejas que promedian los 20mm de largo (Díaz et al. 2011).

Durante los días 20 al 30 de abril de 2015 se realizó una prospección de quirópteros en Bahía Blanca, Fiordo Parry, P.N. Alberto de Agostini (54³5'19.19'S - 6909'28.59'O). Se utilizaron cuatro redes de neblina de 9 metros cada una (M149, ECOTONE, Polonia) para capturar individuos dentro de un bosque maduro de Lenga (Nothofagus pumilio [Poepp. \& Endl.] Krasser). Las redes se abrían al atardecer y permanecían abiertas durante 3 horas aprovechando el período de mayor actividad (Mitchell-jones \& McLeish, 2004; Ossa, 2010), abarcando un esfuerzo de muestreo de 108 metros de red hora / noche. Los individuos capturados fueron medidos con un caliper de precisión $0,01 \mathrm{~mm}$ y una balanza de 50g (PESOLA, Suiza). Para su identificación se utilizó las claves de Bárquez et al. (2009) y Díaz et al. (2011) para luego ser liberados en el sitio de captura.

Se logró capturar cuatro individuos machos adultos de la especie M. chiloensis (Fig. 1). Tres

ConserBat EIRL, Camino a la balsa s/n San Fabián

Región del Biobío, Chile.

$\gg$ chalofoh@mail.com

2 Programa para la Conservación de los murciélagos de Chile (PCMCh). 
individuos fueron capturados a las 19:30 hrs y un individuo fue capturado a las 20:15 hrs. Las medidas morfométricas (promedio (rango)) fueron las siguientes (Tabla 1): peso: 9,9 $(8,5-12,0)$ g; largo total: $81,0(78,3-84,0) \mathrm{mm}$; largo del antebrazo: 38,9 $(38,5-39,3) \mathrm{mm}$; largo del trago: 5,0 (4,8-5,4) mm; ancho del trago: $1,7(1,6-1,9)$ $\mathrm{mm}$; largo de la oreja: $10,4(9,3-11,3) \mathrm{mm}$; ancho de la oreja: $5,5(4,9-5,8) \mathrm{mm}$; largo del 5to dedo: 46,3 (44,1-48,0) mm. Los individuos presentaron una coloración café oscuro, su pelaje presentó un contraste entre la base y las puntas, siendo la base más oscura. Sus orejas son negras y de pequeño tamaño $(<15 \mathrm{~mm})$, lo cual lo diferencia de la especie $H$. magellanicus.

El presente registro de $M$. chiloensis corresponde al primer registro para esta especie en el P.N. Alberto de Agostini y en la Cordillera de Darwin, y dista $100 \mathrm{~km}$ de los registros en la costa norte de Isla Navarino realizados por Koopman (1967). Los individuos capturados presentaron un peso mayor (Tabla 1) que cualquier otro individuos de la especie capturado en Chile (Mann, 1978; Bozinovic et al. 1985; Ossa et al. 2010; Ossa
\& Rodríguez-San Pedro, 2015), esto debido posiblemente a la acumulación de grasa de los individuos capturados en zonas más australes para poder hibernar, dado que no se observan diferencias en estructuras óseas como el largo del antebrazo. Los individuos capturados probablemente utilizan la corteza de árboles adultos para refugiarse dentro del bosque. Dadas las condiciones climáticas extremas en esta zona del país, este registro es valioso al proporcionar nueva información en la distribución de esta especie de murciélago en latitudes sobre los $50^{\circ}$ sur.

La captura de individuos de la especie Myotis chiloensis durante el mes de abril, probablemente indica que estos hibernan en el lugar (Bozinovic et al. 1985) aprovechando las estructuras disponibles en el bosque. Actualmente en Norteamérica los murciélagos del genero Myotis que hibernan a altas latitudes se ven afectados por el síndrome de nariz blanca (WNS) una enfermedad fúngica altamente mortal (Blehert et al. 2009), el estudio del género Myotis en Patagonia es crucial para prevenir una posible infección que afectaría a las poblaciones locales.

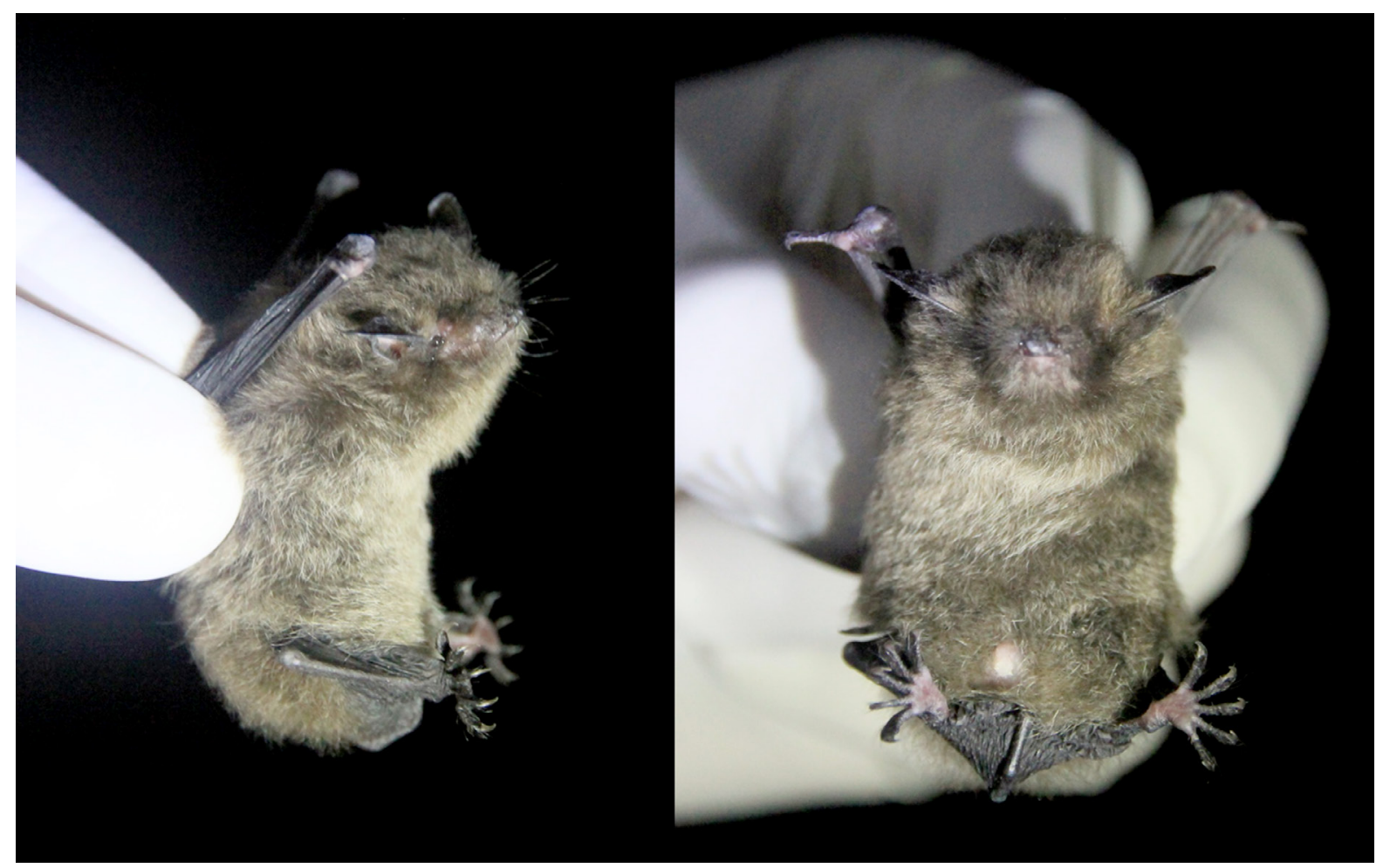

Fig. 1. Individuo macho de a especie Myotis chiloensis capturado en Bahía Blanca, Fiordo Parry, P.N. Alberto de Agostini. 
Tabla 1. Comparativa de las medidas morfométricas de los individuos capturados en la región de Magallanes y otras regiones dentro de la distribución de la especie. Las medidas para las regiones Metropolitana, de La Araucanía y Los Ríos se muestran como (Promedio $\pm \mathrm{DE}$ ); las medidas del presente estudio se muestran como Promedio (Rango). Las referencias para cada región son: Metropolitana: Ossa \& Rodríguez-San Pedro, 2015; La Araucanía: Ossa et al. 2010; Los Ríos: www.sea.gob.cl.

\begin{tabular}{lccccccccc}
\hline \multicolumn{1}{c}{ Región } & $\mathrm{N}$ & Peso (gr) & $\begin{array}{c}\text { Largo total } \\
(\mathrm{mm})\end{array}$ & $\begin{array}{c}\text { Largo del } \\
\text { Antebrazo } \\
(\mathrm{mm})\end{array}$ & $\begin{array}{c}\text { Largo del } \\
\text { Trago }(\mathrm{mm})\end{array}$ & $\begin{array}{c}\text { Ancho del } \\
\text { Trago }(\mathrm{mm})\end{array}$ & $\begin{array}{c}\text { Largo de la } \\
\text { Oreja }(\mathrm{mm})\end{array}$ & $\begin{array}{c}\text { Ancho de } \\
\text { la Oreja } \\
(\mathrm{mm})\end{array}$ & $\begin{array}{c}\text { Largo del } \\
5 \text { to dedo } \\
(\mathrm{mm})\end{array}$ \\
\hline Metropolitana & 23 & $6,2 \pm 2,1$ & $79,3 \pm 3,2$ & $37,0 \pm 1,6$ & $6,0 \pm 0,9$ & $1,7 \pm 0,2$ & $11,5 \pm 1,3$ & $5,9 \pm 0,7$ & $46,1 \pm 1,3$ \\
La Araucanía & 9 & $7,5 \pm 0,9$ & $90,0 \pm 4,2$ & $38,6 \pm 0,8$ & & & $14,5 \pm 1,2$ & \\
Los Ríos & 37 & $7,3 \pm 1,6$ & $79,8 \pm 6,6$ & $37,8 \pm 1,7$ & $5,2 \pm 1,1$ & $1,8 \pm 0,3$ & $11,4 \pm 1,5$ & $6,1 \pm 1,0$ & $44,9 \pm 2,9$ \\
Magallanes & 4 & 9,8 & 81,0 & 38,8 & 5,0 & 1,7 & 10,4 & 5,4 & 46,3 \\
& & $(8,5-12)$ & $(78,3-84,0)$ & $(38,5-39,3)$ & $(4,8-5,4)$ & $(1,6-1,9)$ & $(9,3-11,3)$ & $(4,9-5,8)$ & $(44,1-48,0)$
\end{tabular}

\section{AGRADECIMIENTOS}

El presente estudio fue financiado por el proyecto FONDECYT 1130381. Agradezco a Juan Carlos Aravena del Instituto de la Patagonia por su invitación al extremo sur de Chile, y a quienes nos acompañaron en esta expedición: Scott Reynhout y Rodrigo Soteres.

\section{LITERATURA CITADA}

Bárquez, R., \& Díaz, M. M. (2009). Los murciélagos de Argentina, Clave de identificación. PCMA (Programa de Conservación de los Murciélagos de Argentina), Tucumán, Argentina.

Blehert, D. S., Hicks, A. C., Behr, M., Meteyer, C. U., Berlowski-Zier, B. M., Buckles, E. L., Coleman, J. T. H.,... Stone, W. B. (2009). Bat white-nose syndrome: an emerging fungal pathogen? Science, DOI: 10.1126/ science. 1163874.

Bozinovic, F., Contreras, L. C., Torres-Mura, J., \& Rosenmann, M. (1985). Bioenergética de Myotis chiloensis (Quiróptera: Vespertilionidae). Revista Chilena de Historia Natural, 58, 39-45.

Dabbene, R. (1902). Fauna Magallánica. Mamíferos y aves de la Tierra del Fuego e islas adyacentes. Anales del Museo Nacional de Buenos Aires, 341-409.

Díaz, M. M., Aguirre, L. F., \& Bárquez, R. (2011). Clave de identificación de los murciélagos del cono sur de Sudamérica. Centro de
Estudios de Biología Teórica y Aplicada, Cochabamba, Bolivia.

Giménez, A., Giannini, N. P., Schiffini, M., \& Martin, G. (2012). Records of the rare Histiotus magellanicus (Chiroptera, Vespertilionidae) and other bats from central Patagonia, Argentina. Mastozoología Neotropical, 19, 213-224.

Iriarte, A. (2008). Los mamíferos de Chile. Linx Ediciones, Barcelona, España.

Koopman, K. F. (1967). The southernmost bats. Journal of Mammalogy, 48, 487-488.

Mann, G. (1978). Los pequeños mamíferos de Chile. Gayana Concepción, 40, 1-342.

Martinic, M. (1957). Fauna Magallánica IV los roedores (Macroroedores) y los quirópteros. Boletin Ganadero, 20-22.

Massoia, E., \& Chebez, J. C. (1993). Mamíferos silvestres del archipiélago fueguino. Literature of Latin America (LOLA), Buenos Aires, Argentina.

Mitchell-Jones, A. J., \& McLeish, A. P. (2004). Bat workers manual. Joint Nature Conservation Committee. London, England.

Ossa, G. (2010). Métodos bioacústicos : una aproximación a la ecología de comunidades de murciélagos en las eco-regiones mediterránea y el bosque templado de Chile. Tesis de Ingeniero Agrónomo, Departamento de Zootecnia, Facultad de Agronomía e Ingeniería Forestal, Pontificia Universidad Católica de Chile.

Ossa, G., Ibarra, J. T., Barboza, K., Hernández, F., Gálvez, N., Laker, J., \& Bonacic, C. 
(2010). Analysis of the echolocation calls and morphometry of a population of Myotis chiloensis (Waterhouse, 1838) from the southern Chilean temperate forest. Ciencia Investigación Agraria, 37, 131-139.

Ossa, G., Bonacic, C., \& Bárquez, R. (2014). First record of Histiotus laephotis (Thomas , 1916 ) from Chile and new distributional information for Histiotus montanus (Phillipi and Landbeck, 1861) (Chiroptera, Vespertilionidae ). Mammalia, 79(4), 457461.

Ossa, G., \& Díaz, F. (2014). Histiotus magellanicus (Philippi 1866), un ignorado dentro de la mastofauna chilena. La Chiricoca, 17, 4-6.

Ossa, G., \& Rodríguez-San Pedro, A. (2015).
Myotis chiloensis. Mammalian Species, 47, 51-56.

Rau, J. R., \& Yáñez, J. (1979). Nuevos registros de Lasiurus borealis en Magallanes. Noticiario Mensual del Museo Nacional de Historia Natural, 23, 13-14.

Texera, W. A. (1973). Distribución y diversidad de mamíferos y aves en la provincia de Magallanes. IV. Zoogeografía de mamíferos nativos terrestres. Anales del Instituto de la Patagonia, 4, 321-333.

Venegas, C., \& Sielfeld, W. (1998). Catálogo de los vertebrados de la región de Magallanes y Antártica Chilena. Ediciones de la Universidad de Magallanes, Punta Arenas, Chile. 